\title{
ATP during Early Bladder Stretch Is Important for Urgency in Detrusor Overactivity Patients
}

\author{
Y. Cheng, ${ }^{1}$ K. J. Mansfield, ${ }^{2}$ W. Allen, ${ }^{1}$ R. Chess-Williams, ${ }^{3}$ E. Burcher, ${ }^{4}$ and K. H. Moore \\ ${ }^{1}$ Department of Urogynaecology, St George Hospital, University of New South Wales, Level 1, W.R. Pitney Building, \\ Sydney, NSW 2217, Australia \\ ${ }^{2}$ Graduate School of Medicine, University of Wollongong, Wollongong, NSW 2522, Australia \\ ${ }^{3}$ Faculty of Health Sciences \& Medicine, Bond University, Gold Coast, QLD 4229, Australia \\ ${ }^{4}$ Department of Pharmacology, School of Medical Sciences, University of New South Wales, Sydney, NSW 2052, Australia
}

Correspondence should be addressed to Y. Cheng; y.cheng@unsw.edu.au

Received 28 February 2014; Revised 28 April 2014; Accepted 15 May 2014; Published 27 May 2014

Academic Editor: Michael Winder

Copyright (C) 2014 Y. Cheng et al. This is an open access article distributed under the Creative Commons Attribution License, which permits unrestricted use, distribution, and reproduction in any medium, provided the original work is properly cited.

ATP is an important mediator of urgency in women with detrusor overactivity (DO). In order to understand how different degrees of bladder stretch elicited ATP release in DO patients compared with controls, sequential aliquots were collected during cystometry and ATP release was measured at each degree of bladder filling, in female patients with DO and controls. In both DO and control groups, ATP release was induced during bladder filling, suggesting that stretch stimulated further ATP release. However, the luminal ATP concentrations were already high at early filling stage $(200 \mathrm{~mL})$, which was even greater than those at the later filling stages (400 mL and maximum cystometric capacity, MCC), indicating that a substantial ATP release has been induced during early filling $(200 \mathrm{~mL})$ in both DO and controls. In DO, ATP release at $200 \mathrm{~mL}$ was significantly higher in those with low first desire to void (FDV) $(\leq 200 \mathrm{~mL})$ than in those with higher FDV $(>200 \mathrm{~mL})$; this may suggest that ATP release at early stretch may play an important role in urgency (early sensation) in DO. ATP concentrations remained unchanged after voiding, suggesting that voiding did not further induce ATP release into intraluminal fluid.

\section{Introduction}

The urothelium, which was once regarded as an inert, protective barrier, plays a key role in bladder sensory and motor functions [1]. The hypothesis that the purine nucleotide ATP is a neurotransmitter responsible for nonadrenergic, noncholinergic neurotransmission was proposed in 1972 and termed purinergic signalling [2]. More recently, both animal and human studies show that nonneuronal ATP is released from the bladder urothelium in response to stretch [3-6]. Besides urothelial cells, cultured pig myofibroblasts were also reported to release ATP in response to stretch [3]. In rat bladder, intravesical application of ATP has been shown to induce detrusor overactivity [7].

Urothelial derived mediators, such as acetylcholine and ATP, signal the sensation of bladder fullness to the central nervous system, thus triggering the micturition reflex [8]. Such mediators act upon specific receptors such as P2X receptors located on suburothelial afferent nerves [9], with possible involvement of myofibroblasts [10]. In addition to stretch, ATP is also released from animal bladder urothelial cells and tissue strips in response to capsaicin and acid $[3,5]$. In human subjects, $\mathrm{OAB}$ was shown to be associated with increased ATP release [11, 12].

Previous studies have explored the hypothesis that ATP in bladder luminal fluid could be a marker for the sensation of "urgency" as experienced by patients with overactive bladder symptoms [13, 14]. An inverse correlation between the intravesical concentration of ATP and bladder volume at FDV has previously been reported in two groups: patients with urodynamically proven $\mathrm{DO}[13]$ and patients with clinical $\mathrm{OAB}$ and low FDV [14]. However, the mechanism responsible for this was not elucidated.

Stretch is considered to be a major stimulus for ATP release in the bladder and it was hypothesized therefore that more ATP should be released as the bladder fills, with the 
highest ATP levels occurring at the end of the filling stage in accordance with murine studies which showed increased ATP release with increased intravesical pressure [15]. However, to our knowledge there are no reports indicating exactly when ATP is released during human bladder filling or how ATP release is influenced by the degree of bladder stretch. Therefore, we performed cystometry in women, collecting consecutive samples of bladder fluid at $200 \mathrm{~mL}$ and $400 \mathrm{~mL}$ and MCC for sequential ATP measurement. We recruited control patients and those with DO in order to characterise and compare the pattern of sequential ATP release in these two groups.

\section{Materials and Methods}

2.1. Cystometry. Each female patient was catheterised to empty the bladder completely for routine clinical cystometry [16], with the exception that dual lumen catheters were employed. A CSU was sent for culture and patients with proven bacterial cystitis ("UTI" $10^{8} \mathrm{cfu} / \mathrm{L}$ with pyuria $>10 / \mathrm{HPF}$ ) were excluded from subsequent analysis. Female patients gave informed consent in accordance with approval from the South Eastern Sydney human research ethics committee (SESIAHS HREC 06/11).

Saline at room temperature (approximately $25^{\circ} \mathrm{C}$ ) was infused into the bladder at a filling rate of $75 \mathrm{~mL} / \mathrm{min}$ (medium-fill) [16] as described previously [13]. As per routine, volumes were noted when the patient felt FDV and reached maximum cystometric capacity (MCC), (the volume at which the patient feels she can no longer delay micturition [16]), when filling was stopped. Tap water stimuli and erect provocation were routinely performed. At the end of cystometry, the patient voided the filling solution into a clean uroflow chamber, in private. The presence of any detrusor contractions during filling, or provocation such as the sound of running tap water or changing supine to erect position, was noted.

Patients were characterised as (a) idiopathic DO, with involuntary detrusor contractions during the filling phase which may be spontaneous or provoked, or (b) control, that is, pure urodynamic stress incontinence, involuntary leakage of urine during increased abdominal pressure in the absence of detrusor contractions, and no symptoms of urgency. Patients with the following were excluded: (a) bladder pain syndrome (FDV < $200 \mathrm{~mL}$ with bladder pain, and MCC < $400 \mathrm{~mL}$ ), (b) voiding dysfunction (detrusor pressure (Pdet) at maximal flow greater than $60 \mathrm{~cm} \mathrm{H}_{2} \mathrm{O}$ ), (c) incomplete voiding (postvoid residual volume $>100 \mathrm{~mL}$ ), or (d) evidence of spinal cord or central neurological disease.

2.2. Sampling and ATP Measurements. When the filling volume reached $200 \mathrm{~mL}, 400 \mathrm{~mL}$, and $\mathrm{MCC}$, the infusion was paused for sample collection. These volumes were chosen as fixed values that approximate the commonly observed filling volumes for first desire to void and maximal capacity in patients with DO (see discussion). At each volume, the first $10 \mathrm{~mL}$ in the collecting catheter ("deadspace") was aspirated and discarded; then, a further $5 \mathrm{~mL}$ of intravesical filling fluid was collected. After voiding, fluid from the uroflow chamber was collected (known as "voided volume," VV). Each woman thus yielded four samples for ATP assay. Immediately after collection, samples were transported on ice from the clinic to the laboratory in the adjacent building and then ATP assayed immediately.

ATP concentration (in $\mathrm{nM}$ ) was measured in duplicate per sample, using a GloMax 20/20 luminometer and a bioluminescence assay (Sigma). A standard curve was constructed using freshly made standards $\left(10^{-5}\right.$ to $10^{-10} \mathrm{M}$ and blank) from frozen concentrated ATP stock. The total amount of ATP (in nmoles) contained in each sample was then calculated by multiplying the filling volume $(200 \mathrm{~mL}, 400 \mathrm{~mL}$, $\mathrm{MCC}$, or VV) by its corresponding ATP concentration.

2.3. Statistical Analysis. ATP data were not normally distributed $(P<0.05$, D’Agostino \& Pearson omnibus normality test) and were expressed as median (interquartile range, IQR). Nonparametric statistical analyses were performed using GraphPad Prism 6.0 software (San Diego, USA). Comparison of ATP levels in the different samples was performed by Friedman ANOVA with Dunn's multiple comparisons test (Figures 1 and 4) or using a Mann-Whitney test when two unmatched groups were compared (Figure 2) or a Wilcoxon test when matched groups were compared (Figure 3). Methods, definitions, and units conform to the standards recommended by the International Continence Society except where specifically noted.

\section{Results and Discussions}

3.1. Stretching Is a Stimulate to Induce Further ATP Release in Both DO and Controls. Although ATP release is induced by urothelial stretch during bladder filling, it is unclear whether stretch-induced ATP is involved in triggering bladder contraction, either at physiological maturation or during pathological conditions such as DO, and the pattern of ATP release in human bladder and its association with bladder sensation have not been clearly understood. Traditional animal models cannot provide this information, because of the small bladder volume in rats and mice and the inability to assess the sensations of bladder filling in the rodent. Results from in vitro laboratory studies reporting ATP release from human bladder urothelium/lamina propria strips have shown a very marked increase in stretch-induced ATP release in DO, compared with control [12]. Using urodynamic fluid to investigate ATP release has become a valuable tool to answer these questions under true physiological condition relevant to patients.

To investigate how bladder stretch could induce ATP release, we also chose to collect samples at volumes of $200 \mathrm{~mL}$, which approximates the FDV in most patients (median FDV in DO $167 \mathrm{~mL}$ and control $200 \mathrm{~mL}$, Table 1), and a filling volume of $400 \mathrm{~mL}$, which is close to the MCC (median MCC $430 \mathrm{~mL}$ ) in most DO patients, in addition to sampling at MCC and in voided fluid. We compared ATP release in these consecutive intraluminal fluid samples from each patient. These intraluminal fluid samples have been generated during 


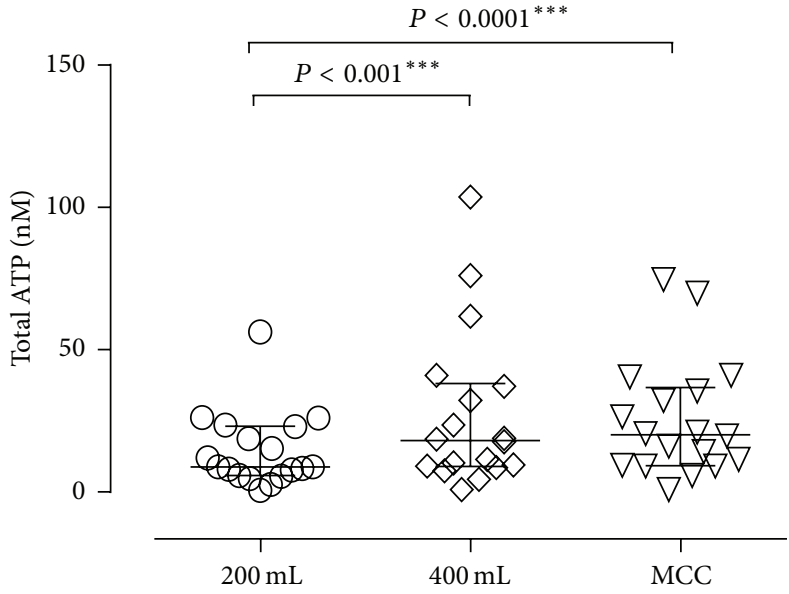

(a)

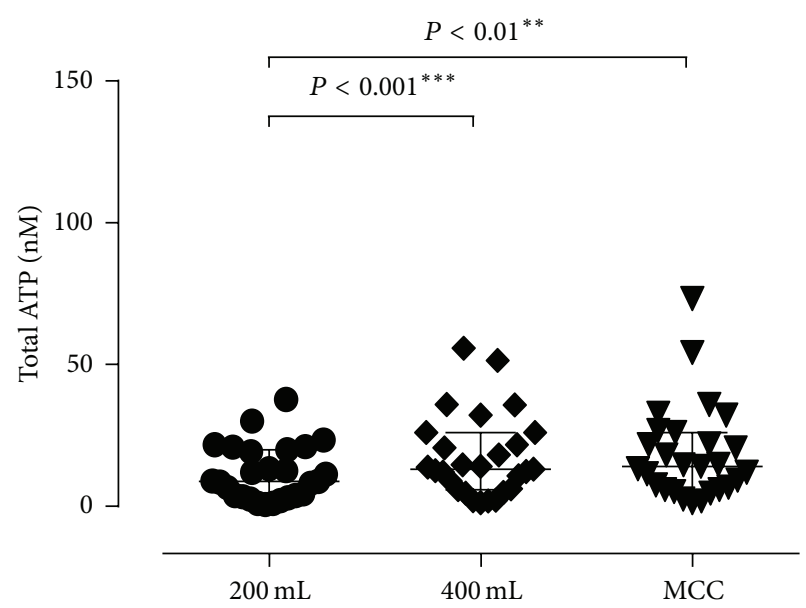

(b)

Figure 1: Comparison of total ATP (nmoles) in bladder fluid collected at volumes of $200 \mathrm{~mL}$ and $400 \mathrm{~mL}$ and at MCC, in control $(n=18)$ (a) and DO $(n=27)$ (b). ATP content significantly increased between filling volumes of $200 \mathrm{~mL}$ and $400 \mathrm{~mL}$ and between $200 \mathrm{~mL}$ and MCC. Data were analyzed by the Friedman test followed by Dunn's multiple comparison test. $\left({ }^{* *}=P<0.01\right.$; $\left.^{* * *}=P<0.001\right)$.

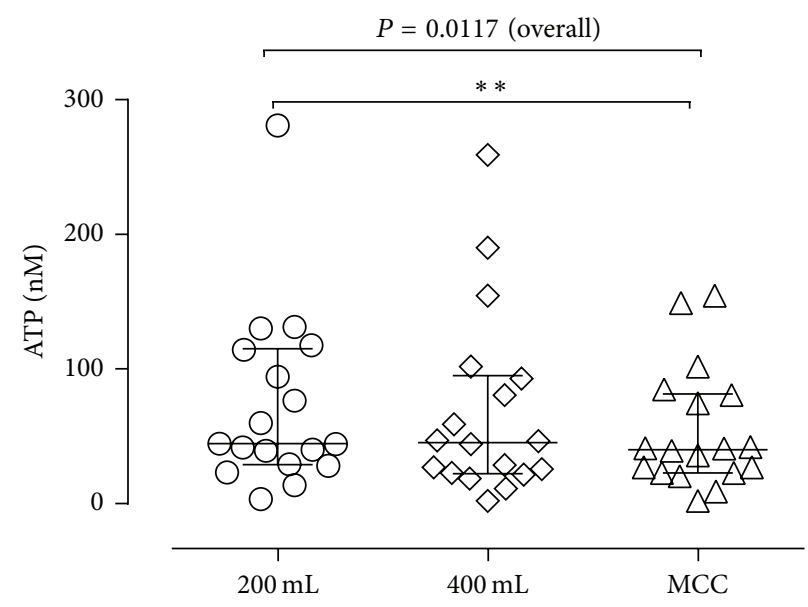

(a)

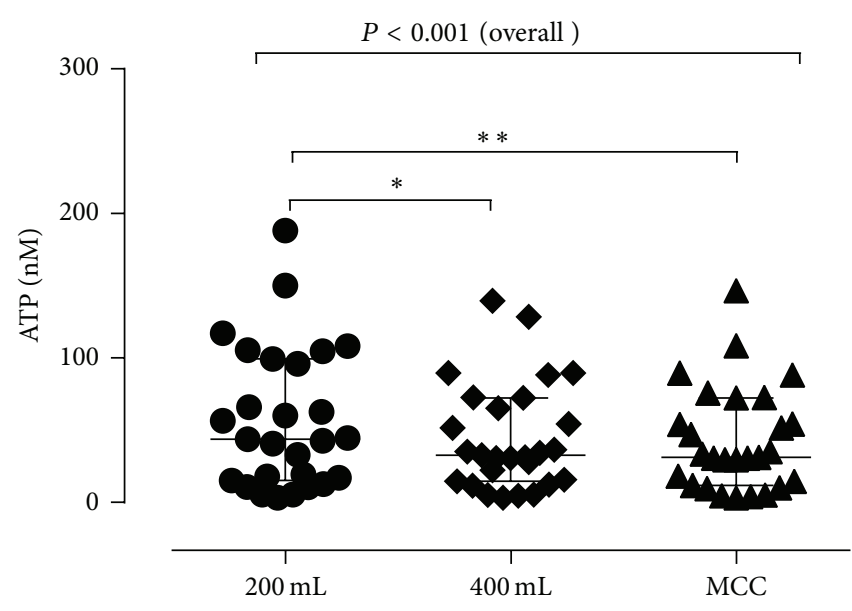

(b)

FIGURE 2: Comparison of ATP concentrations in bladder fluid collected at volumes of $200 \mathrm{~mL}$ and $400 \mathrm{~mL}$ and at MCC for control ( $n=18$ ) (a) and DO $(n=27)$ (b) patients. In this and subsequent figures, data points are from individual patients, showing median and interquartile range. Data were analyzed by the Friedman test followed by Dunn's multiple comparison test.

TABle 1: Patient urodynamic characteristics.

\begin{tabular}{lcr}
\hline & Control (median (IQR)) & DO (median (IQR)) \\
\hline$n$ & 18 & 27 \\
Age (years) & $60.5(47-70)$ & $59(54-66)$ \\
FDV $(\mathrm{mL})$ & $200(170-265)$ & $167(100-277)$ \\
MCC $(\mathrm{mL})$ & $492(450-500)$ & $430(400-500)$ \\
${ }^{*}$ Max Pdet during filling $\left(\mathrm{cm} \mathrm{H}_{2} \mathrm{O}\right)$ & $6.5(5-9.25)$ & $33(17-46)$ \\
Detrusor contractions during cystometry & 0 & 27 \\
VV $(\mathrm{mL})$ & $485(415-532.5)$ & $417(380-500)$
\end{tabular}

${ }^{*}$ Maximal detrusor pressure during filling or provocation. 


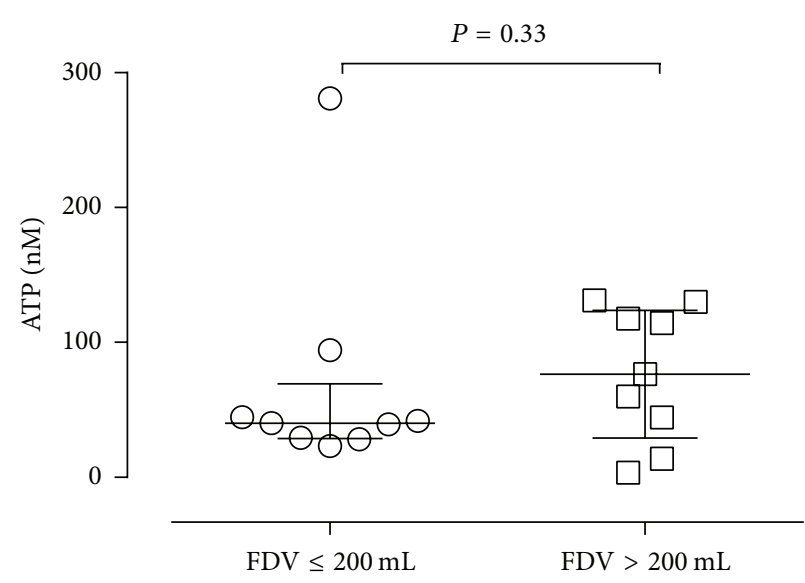

(a)

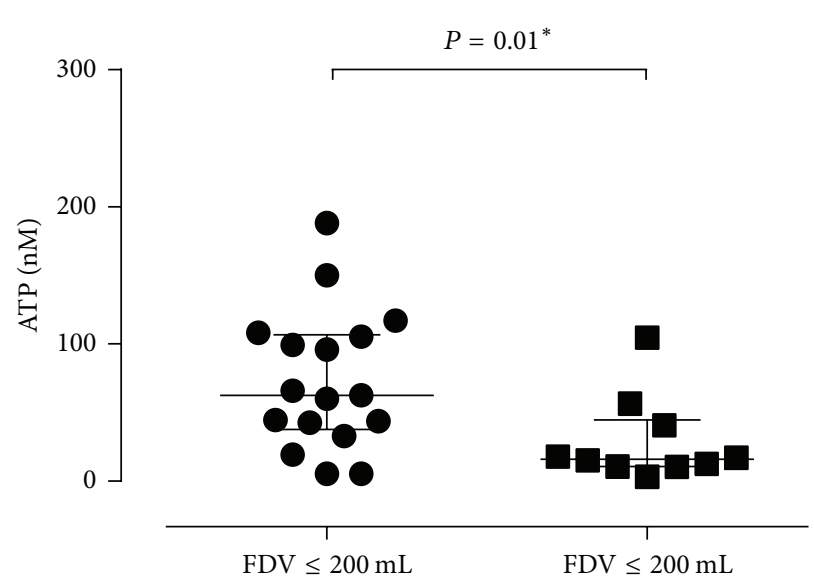

(b)

FIGURE 3: Comparison of ATP release at a bladder volume of $200 \mathrm{~mL}$. For control (a) and DO patients (b), the data have been divided into two groups of low $(\leq 200 \mathrm{~mL})$ and higher FDV $(>200 \mathrm{~mL})$. The two control groups of low $(n=9)$ and high $(n=9)$ FDV showed no significant difference $(P=0.33)$, whereas there was a significantly higher concentration of ATP in the DO group with low FDV $(n=17)$, compared to the DO group with higher FDV $(n=10)(P=0.01$, Mann-Whitney test).

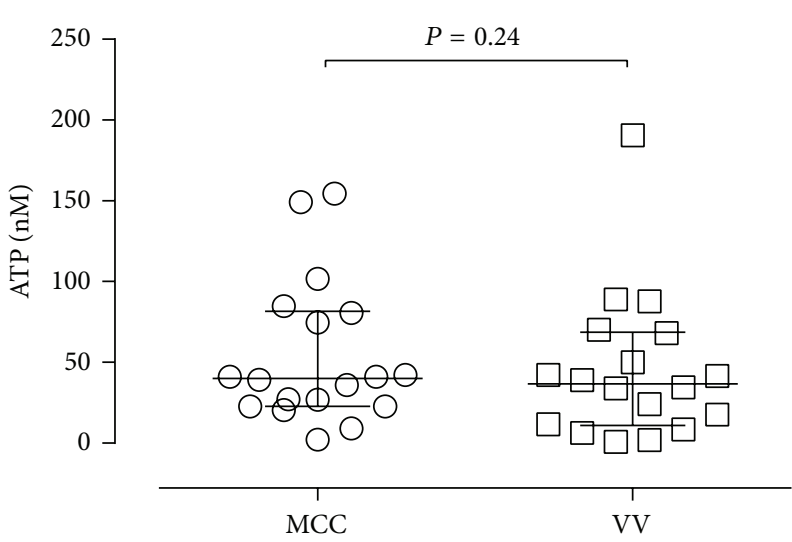

(a)

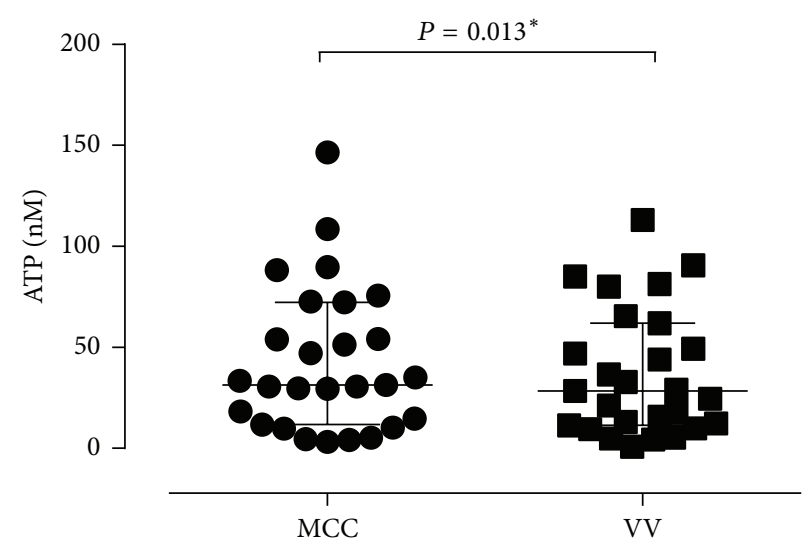

(b)

FIGURE 4: Comparison of ATP concentration before and after voiding in (a) control $(n=18)$ and (b) DO patients $(n=27)$. For control patients, there was no difference in ATP concentration of urodynamic fluid before MCC or after voiding (VV). For DO patients, there was a significant decrease in ATP concentration in VV compared with MCC $(P=0.01$, Wilcoxon test).

routine urodynamic testing under standard clinical conditions which occurs at a supraphysiological filling rate $(75 \mathrm{~mL} /$ $\min )[16]$.

Cystometry with measurement of ATP at the four filling volumes was performed on 53 women (age 28-87 yrs). Eight subjects with UTI on the test day and 2 subjects with voiding dysfunction were excluded, yielding 27 patients with DO and 18 control patients (Table 1).

The total amount of ATP (in nmoles) at each bladder volume was examined. For both control (Figure 1(a)) and DO (Figure 1(b)), there was a significant increase in the total amount of ATP, in nmoles, between $200 \mathrm{~mL}$ and $400 \mathrm{~mL}$, as well as between $200 \mathrm{~mL}$ and MCC; the total amount of ATP in the intraluminal fluid (in nmoles) was the lowest at the lowest bladder filling volume (total ATP median values (in nmoles) at $200 \mathrm{~mL}, 400 \mathrm{~mL}$, and MCC are 8.8,13.1, and 14, for DO, and 8.9, 18.1, and 20.2, for controls). Note that ATP was released continuously throughout the urodynamic test in both DO and control and that the total ATP content in the intraluminal fluid increased during filling, that is, from $200 \mathrm{~mL}$ to $400 \mathrm{~mL}$, which agreed with the initial hypothesis that ATP release was induced by bladder stretch in both DO and controls.

We have also compared the difference in ATP release between DO and control groups at each filling/sampling point, but we did not see any significant difference between the two groups. One of the reasons for this could be the variations in ATP release in individuals, which made it too hard to see the difference between two groups. This is not 
a surprise as there was no significant difference in ATP release between DO and control patients with a much larger sample size in a previous study [13].

\subsection{Substantial ATP Released at the Early Bladder Filling} Stage. We also compared the ATP concentrations according to the filling volumes and determined that in both control (Figure 2(a)) and DO (Figure 2(b)) patients, there was a significant difference in ATP concentration at the three different bladder volumes (Friedman test). Overall, in both control and DO patients the ATP concentration was higher at the early filling stage $(200 \mathrm{~mL}$, median ATP $44.5 \mathrm{nM}$ control; $43.8 \mathrm{nM}$ DO) compared with later stages (MCC, median ATP 40.2 control; 31.3.7 nM DO).

This is a surprising and important finding. This indicates that there was substantial ATP release at early filling volumes in both DO and controls, and early ATP release may play an important role in bladder function and dysfunction, that is, early sensation or urgency for DO.

\subsection{Early ATP Concentration Is Related to the First Desire Vol-} ume (FDV) in DO Patients. In the DO patients, the median FDV was $167 \mathrm{~mL}$, with values ranging between 85 and $400 \mathrm{~mL}$. In order to investigate whether FDV was associated with ATP release, patients were divided into two groups based on low FDV $(\leq 200 \mathrm{~mL})$ and higher FDV $(>200 \mathrm{~mL})$. In control patients, no difference in ATP concentration in intravesical fluid at $200 \mathrm{~mL}$ was seen between the two groups (FDV less than $200 \mathrm{~mL}$ (ATP median $=40.2 \mathrm{nM}$ ) and FDV more than $200 \mathrm{~mL}($ ATP median $=76.5 \mathrm{nM})$ ) (Figure 3(a)). However, in DO patients, the concentration of ATP in intravesical fluid at $200 \mathrm{~mL}$ was significantly higher in patients with FDV $\leq$ $200 \mathrm{~mL}$ than those with FDV > $200 \mathrm{~mL}$ (Figure 3(b)). The same analysis was applied to the data sets from the $400 \mathrm{~mL}$, MCC, and VV samples, but no association was shown between FDV and ATP release at these volumes (data not shown).

The fact that DO patients with a lower FDV $(\leq 200 \mathrm{~mL})$ had higher ATP concentrations (ATP median $62.7 \mathrm{nM}$ ) at an earlier bladder volume, compared with DO patients who had a higher FDV (>200 mL) (ATP median $16.1 \mathrm{nM})$, suggests that the early ATP release may play an important role in DO patients, which may cause urgency. The previous clinical studies have also found that ATP in voided urodynamic fluid was inversely correlated with the FDV in DO [13] and OAB [14] patients, but not controls. This current study supports the supposition that it is the early ATP release which is important to the sensation of urgency in DO patients.

Although ATP can be degraded by membrane-bound ectoATPases, studies have shown that there is little ectoATPase activity associated with the apical surface of the urothelium [17]; thus the intravesical ATP measured may reflect the ATP released from the urothelial cells. Moreover, the source of the ATP is assumed to be from the urothelial cells, but this was not able to be determined from our in vivo study. The ATP in bladder intraluminal fluid might be released directly from urothelial cells into the lumen and/or could diffuse into the lumen from various sources within the bladder wall, such as from the suburothelial myofibroblasts. Diffusion into the lumen from suburothelial cells would be limited by the intact bladder urothelium and also by the ectoATPase activity associated with intermediate and basal urothelial cells and also with the lamina propria [17]. However, a reduced ectoATPase activity in laboratory samples of OAB has been reported [18]; thus, more ATP may diffuse into deep sites of bladder wall, enhancing stretch-induced signalling pathways inducing urgency or involuntary contractions. These findings may explain why the early ATP release plays an important role in early sensation (urgency) in DO but not in controls.

3.4. Voiding Did Not Further Induce ATP Release. Previous studies have reported that ATP plays a role in the bladder as a purinergic neurotransmitter in functional motor [6] as well as sensory bladder disorders [12]. Thus ATP might also appear in the bladder as a result of parasympathetic nerve stimulation to the detrusor during voiding [19].

In order to investigate whether a micturition contraction might increase ATP release in the bladder, we compared the ATP concentration before voiding (MCC) and after voiding (VV). The results showed no significant difference in ATP concentration between MCC (ATP median $=40.2 \mathrm{nM})$ and voided volumes (ATP median $=36.8 \mathrm{nM}$ ) in control patients (Figure 4(a)); that is, ATP content remained unchanged after voiding. In DO patients (Figure 4(b)), there was no evidence of any increase in ATP release and in fact the ATP concentration was slightly lower in voided fluid (VV) (ATP median $=28.4 \mathrm{nM})$ than in bladder intraluminal fluid at MCC $($ ATP median $=31.3 \mathrm{nM})(P=0.01$, Wilcoxon test $)$. This suggests that the voiding contraction does not contribute further to intravesical ATP release in bladder lumen.

This is not surprising as ATP released from motor nerves will be deep in the detrusor muscle layer and unlikely to reach the bladder lumen due to abundant ecto-ATPase in lamina propria and smooth muscle [17]. Instead of increased ATP after voiding, we have noticed a decreased intravesical ATP after voiding in DO patients in our study. It is possible that, during micturition, the intravesical ATP is exposed to ectoATPase located on exfoliated urothelial cells [20] and, therefore, ATP may undergo enhanced degradation during or after voiding.

\section{Conclusions}

For the first time ATP release during bladder filling has been investigated in sequential intraluminal fluid samples in female patients with or without DO. This study has shown that total amount of ATP in intraluminal fluid (in nmoles) continued to increase from lower bladder volume $(200 \mathrm{~mL})$ to higher bladder volume (400 mL and MCC) in both DO and controls. The study demonstrates that stretch (bladder filling) can induce further ATP release in human bladder. Interestingly, we have noticed that there was a substantial ATP release in intraluminal fluid in the early filling stage, that is, bladder filling volume of $200 \mathrm{~mL}$. The results also show that ATP release at $200 \mathrm{~mL}$ filling volume is related to FDV only in DO patients but this relationship was not observed in the control group. 
These data indicate that ATP release at early bladder filling may play an important role in increased bladder sensations, for example, urgency, in DO patients.

\section{Abbreviations}

ATP: Adenosine triphosphate

CSU: Catheter specimen of urine

DO: Detrusor overactivity

FDV: First desire to void

MCC: Maximal cystometric capacity

Pdet: Maximal detrusor pressure

VV: Voided volume.

\section{Conflict of Interests}

All authors have no conflict of interests regarding this paper.

\section{Acknowledgments}

The authors thank Dr. Colin Walsh and Ann Siddons RN for helping with patient recruitment and cystometry testing. This study was supported by the National Health and Medical Research Council of Australia (ID527502).

\section{References}

[1] L. A. Birder, A. J. Kanai, F. Cruz, K. Moore, and C. H. Fry, "Is the urothelium intelligent?" Neurourology and Urodynamics, vol. 29, no. 4, pp. 598-602, 2010.

[2] G. Burnstock, "Purinergic nerves," Pharmacological Reviews, vol. 24, no. 3, pp. 509-581, 1972.

[3] Y. Cheng, K. J. Mansfield, S. L. Sandow, P. Sadananda, E. Burcher, and K. H. Moore, "Porcine bladder urothelial, myofibroblast, and detrusor muscle cells: characterization and ATP release," Frontiers in Pharmacology, vol. 2, article 27, 2011.

[4] D. R. Ferguson, I. Kennedy, and T. J. Burton, "ATP is released from rabbit urinary bladder epithelial cells by hydrostatic pressure changes-a possible sensory mechanism?" Journal of Physiology, vol. 505, no. 2, pp. 503-511, 1997.

[5] P. Sadananda, F. Shang, L. Liu, K. J. Mansfield, and E. Burcher, "Release of ATP from rat urinary bladder mucosa: role of acid, vanilloids and stretch," The British Journal of Pharmacology, vol. 158, no. 7, pp. 1655-1662, 2009.

[6] V. Kumar, C. C. Chapple, and R. Chess-Williams, "Characteristics of adenosine triphosphatase release from porcine and human normal bladder," Journal of Urology, vol. 172, no. 2, pp. 744747, 2004.

[7] J. Nishiguchi, Y. Hayashi, M. B. Chancellor et al., "Detrusor overactivity induced by intravesical application of adenosine $5^{\prime}$ triphosphate under different delivery conditions in rats," Urology, vol. 66, no. 6, pp. 1332-1337, 2005.

[8] L. Birder and K.-E. Andersson, "Urothelial signaling," Physiological Reviews, vol. 93, no. 2, pp. 653-680, 2013.

[9] D. A. Cockayne, S. G. Hamilton, Q. M. Zhu et al., "Urinary bladder hyporeflexia and reduced pain-related behaviour in P2X3deficient mice," Nature, vol. 407, no. 6807, pp. 1011-1015, 2000.

[10] C. Wu, G. P. Sui, and C. H. Fry, "Purinergic regulation of guinea pig suburothelial myofibroblasts," Journal of Physiology, vol. 559, no. 1, pp. 231-243, 2004.
[11] V. Kumar, C. R. Chapple, A. M. Surprenant, and R. Chess-Williams, "Enhanced adenosine triphosphate release from the urothelium of patients with painful bladder syndrome: a possible pathophysiological explanation," Journal of Urology, vol. 178, no. 4, pp. 1533-1536, 2007.

[12] V. Kumar, C. R. Chapple, D. Rosario, P. R. Tophill, and R. ChessWilliams, "In vitro release of adenosine triphosphate from the urothelium of human bladders with detrusor overactivity, both neurogenic and idiopathic," European Urology, vol. 57, no. 6, pp. 1087-1092, 2010.

[13] Y. Cheng, K. J. Mansfield, W. Allen, C. A. Walsh, E. Burcher, and K. H. Moore, "Does adenosine triphosphate released into voided urodynamic fluid contribute to urgency signaling in women with bladder dysfunction?" Journal of Urology, vol. 183, no. 3, pp. 1082-1086, 2010.

[14] Y. Cheng, K. J. Mansfield, W. Allen, R. J. Millard, E. Burcher, and K. H. Moore, "Correlation between cystometric volumes, ATP release, and $\mathrm{pH}$ in women with overactive bladder versus controls," Neurourology and Urodynamics, vol. 32, no. 7, pp. 969973, 2013.

[15] M. Vlaskovska, L. Kasakov, W. Rong et al., "P2X3 knock-out mice reveal a major sensory role for urothelially released ATP," Journal of Neuroscience, vol. 21, no. 15, pp. 5670-5677, 2001.

[16] P. Abrams, L. Cardozo, M. Fall et al., "The standardisation of terminology of lower urinary tract function: report from the standardisation sub-committee of the international continence society," Neurourology and Urodynamics, vol. 21, no. 2, pp. 167178, 2002.

[17] W. Yu, S. C. Robson, and W. G. Hill, "Expression and distribution of ectonucleotidases in mouse urinary bladder," PLOS ONE, vol. 6, no. 4, Article ID e18704, 2011.

[18] R. A. Harvey, D. E. Skennerton, D. Newgreen, and C. H. Fry, "The contractile potency of adenosine triphosphate and ectoadenosine triphosphatase activity in guinea pig detrusor and detrusor from patients with a stable, unstable or obstructed bladder," Journal of Urology, vol. 168, no. 3, pp. 1235-1239, 2002.

[19] G. Burnstock, "Therapeutic potential of purinergic signalling for diseases of the urinary tract," BJU International, vol. 107, no. 2, pp. 192-204, 2011.

[20] J. Southgate, C. L. Varley, M. A. Garthwaite et al., "Differentiation potential of urothelium from patients with benign bladder dysfunction," BJU International, vol. 99, no. 6, pp. 1506-1516, 2007. 


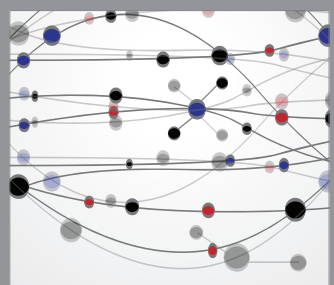

The Scientific World Journal
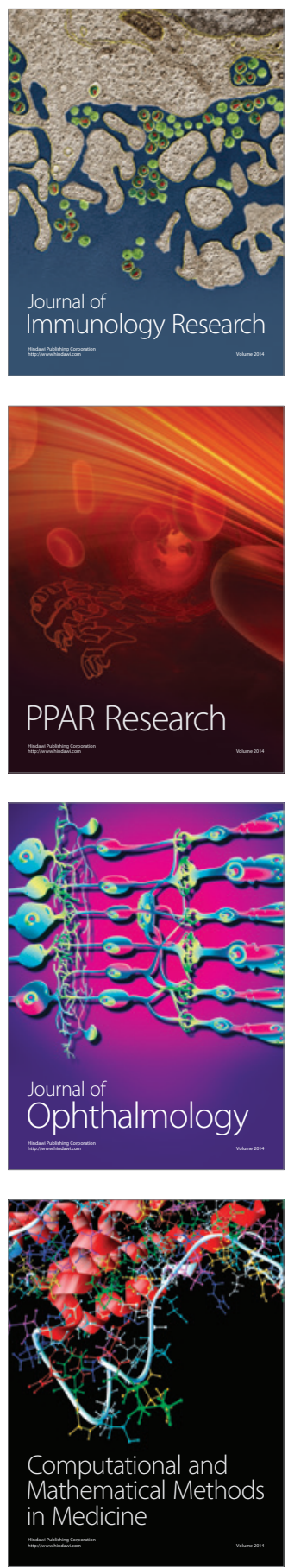

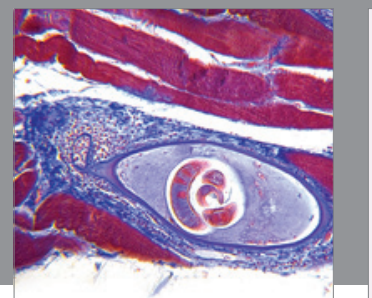

Gastroenterology

Research and Practice
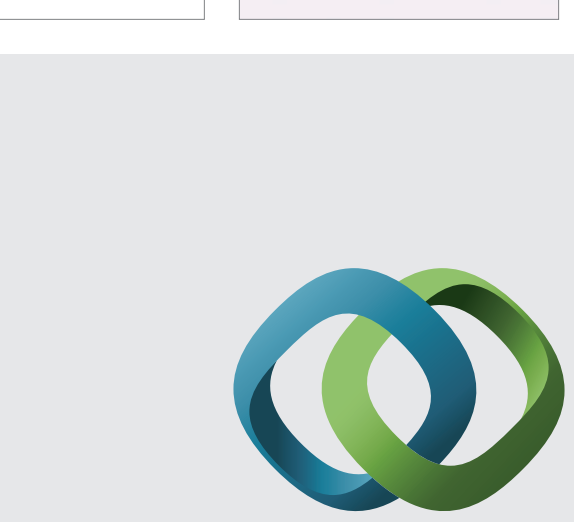

\section{Hindawi}

Submit your manuscripts at

http://www.hindawi.com
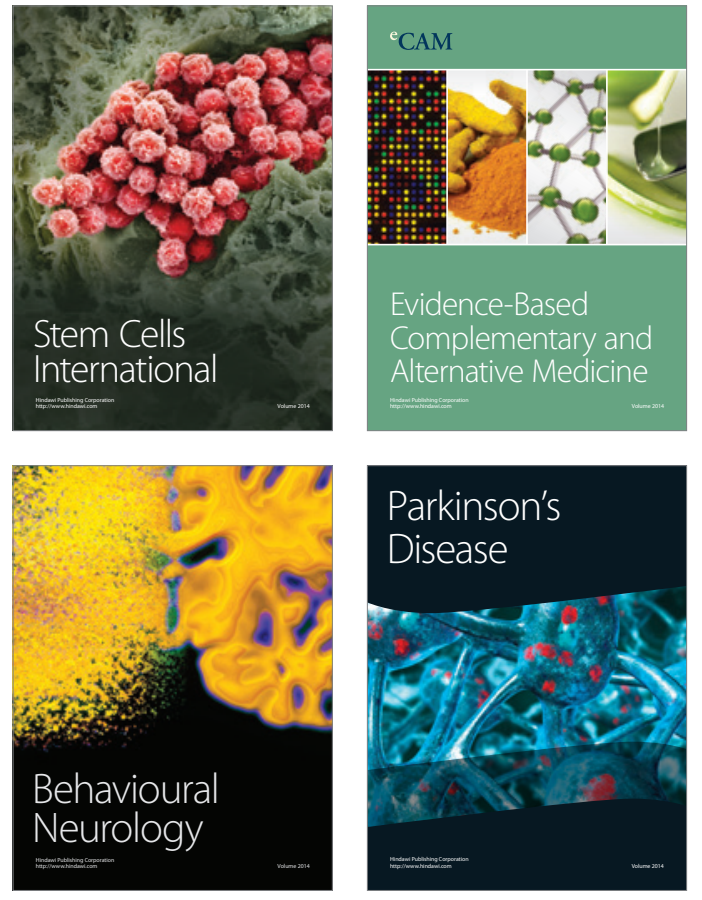
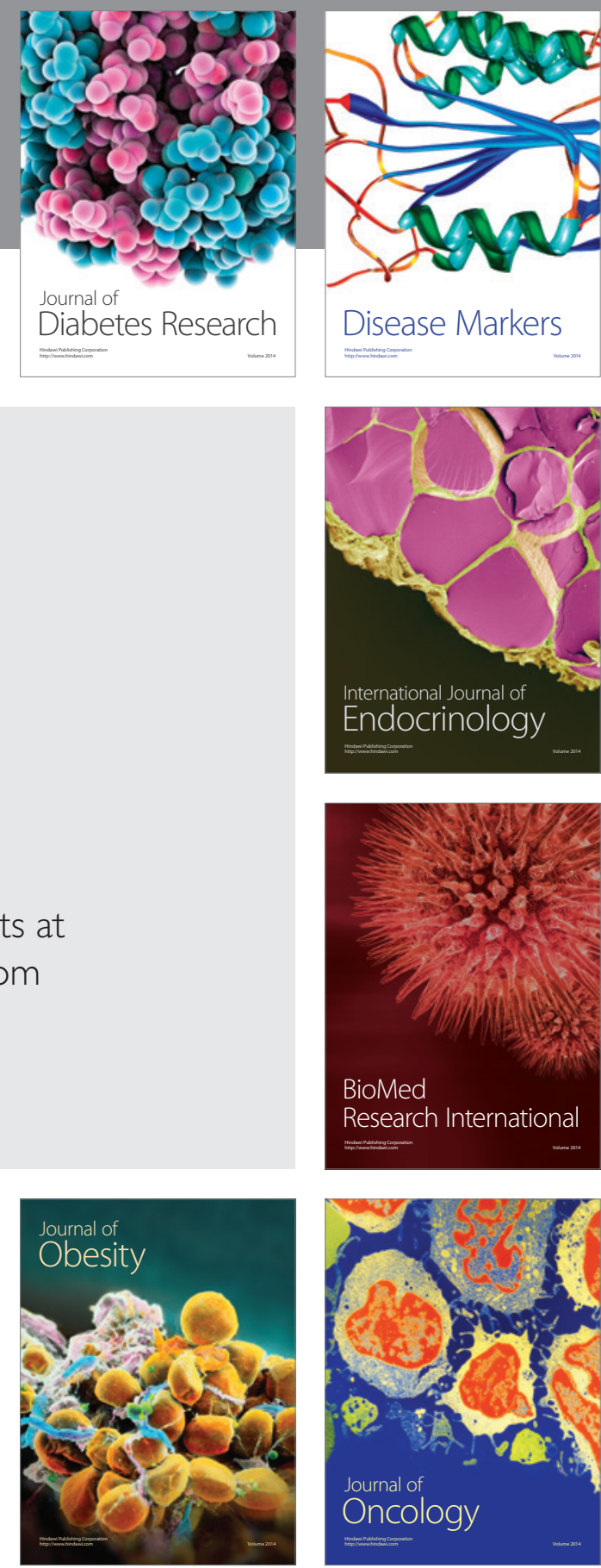

Disease Markers
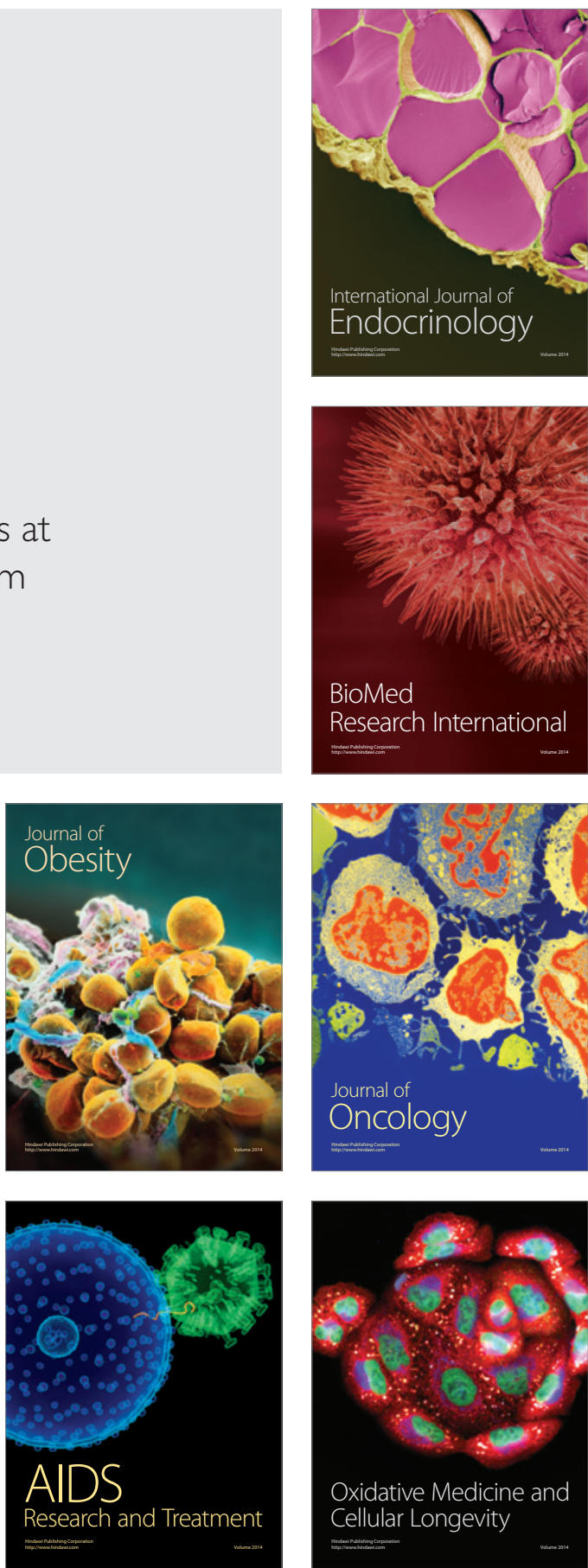